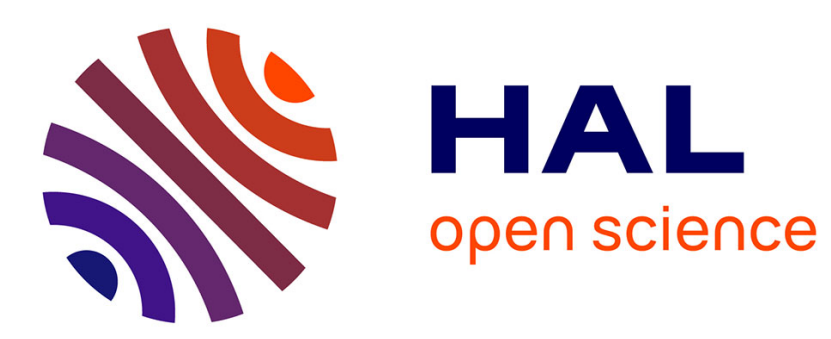

\title{
Stability Notions for a Class of Nonlinear Systems with Measure Controls
}

Aneel Tanwani, Bernard Brogliato, Christophe Prieur

\section{To cite this version:}

Aneel Tanwani, Bernard Brogliato, Christophe Prieur. Stability Notions for a Class of Nonlinear Systems with Measure Controls. Mathematics of Control, Signals, and Systems, 2015, 27 (2), pp.245275. 10.1007/s00498-015-0140-7 . hal-01074144

\section{HAL Id: hal-01074144 https://hal.inria.fr/hal-01074144}

Submitted on 23 Feb 2015

HAL is a multi-disciplinary open access archive for the deposit and dissemination of scientific research documents, whether they are published or not. The documents may come from teaching and research institutions in France or abroad, or from public or private research centers.
L'archive ouverte pluridisciplinaire HAL, est destinée au dépôt et à la diffusion de documents scientifiques de niveau recherche, publiés ou non, émanant des établissements d'enseignement et de recherche français ou étrangers, des laboratoires publics ou privés. 


\title{
Stability Notions for a Class of Nonlinear Systems with Measure Controls
}

\author{
Aneel Tanwani • Bernard Brogliato . \\ Christophe Prieur
}

the date of receipt and acceptance should be inserted later

\begin{abstract}
We consider the problem of stability in a class of differential equations which are driven by a differential measure associated with inputs of locally bounded variation. After discussing some existing notions of solution for such systems, we derive Lyapunov-based conditions on the system's vector fields for asymptotic stability under a specific class of inputs. These conditions are based on the stability margin of the Lebesgue-integrable and the measure-driven components of the system. For more general inputs which do not necessarily lead to asymptotic stability, we then derive conditions such that the maximum norm of the resulting trajectory is bounded by some function of the total variation of the input, which generalizes the notion of integral input-to-state stability in measure-driven systems.
\end{abstract}

Keywords Measure differential equations; measure controls; nonlinear systems; asymptotic stability; input-to-state stability with variation; Lyapunov analysis

\section{Introduction}

In the theory of dynamical systems, a large class of nonlinear systems are modeled by the following ordinary differential equation (ODE):

$$
\frac{d x}{d t}(t)=f(x(t))+g(x(t)) u(t)
$$

where $f, g: \mathbb{R}^{n} \rightarrow \mathbb{R}^{n}$ are vector fields and $u:\left[t_{0}, \infty\right) \rightarrow \mathbb{R}$ denotes the input. If $f(\cdot)$ and $g(\cdot)$ are locally Lipschitz then there exists an interval over which system (1) admits a unique absolutely continuous solution $x(\cdot)$ for every locally essentially bounded Lebesgue-measurable function $u(\cdot)$, such that the resulting solution satisfies (1) almost everywhere (with respect to the Lebesgue

A. Tanwani · B. Brogliato

Team BipOp, Institut National de Recherche en Informatique et Automatique (INRIA), Rhône-Alpes, 655 Avenue de l'Europe, 38334 Montbonnot Cedex, France. E-mail: \{aneel.tanwani, bernard.brogliato\}@inria.fr

C. Prieur

Gipsa-lab, Department of Automatic Control, 11 Rue des Mathématiques, BP 46, 38402 Saint Martin d'Hères, France. E-mail: christophe.prieur@gipsa-lab.fr.

This work has been partially supported by the LabEx PERSYVAL-Lab (ANR-11-LABX0025-01) 
measure) on the interval where the solution exists. Under further regularity assumptions, say $f(\cdot), g(\cdot)$ satisfy a linear growth condition, the solution is defined for all times, in which case we say that $f(\cdot), g(\cdot)$ are forward complete.

In this paper, we consider measure differential equations (MDEs) modeled as:

$$
d x=f(x) d t+g(x) d u .
$$

Just like (1) is driven by an input $u(\cdot)$, the dynamics of system (2) can be seen as driven by the "derivative" of $u(\cdot)$. However, to achieve generality and model discontinuous trajectories, we assume that $u(\cdot)$ is a right-continuous function with locally bounded variation (locally $R C B V$ ), so its generalized derivative, or the so-called differential measure, is represented by $d u$ (see Section 2 for terminology). If $u(\cdot)$ is absolutely continuous, then the derivative of $u(\cdot)$ is a locally essentially bounded Lebesgue-measurable function. In that case, we take $d u=\dot{u} d t$, where $\dot{u}(\cdot)$ represents the classical derivative, and system (2) is equivalent to (1) with $u(\cdot)$ replaced by $\dot{u}(\cdot)$.

\subsection{Literature Overview}

The study of dynamical systems driven by measure first appeared in the context of optimal control problems where the constraint on the control input is that its integral remains bounded without any bounds on the supremum norm $[34,36,42]$. Such optimization problems do not possess a solution when searching for the control input in the class of Lebesgue-measurable functions, and hence the motivation to use measures as control input arises; we discuss such an example in Section 1.3. A practical example of such optimal control problem includes controlling the position of spacecraft with minimum fuel expenditure with no constraints on the thrust vector [31]. More recently, optimization problems over the space of measures have been treated in [26, 32, 37] for nonlinear systems. The work of [5] studies measure-driven control systems with some applications to mechanics. In [8], and references therein, it is seen that the first order necessary conditions for the optimization of a quadratic cost problem subjected to systems with unilateral constraints result in differential equations with measures. Other than the optimization problems, control of mechanical systems in the presence of friction may necessitate the use of impulsive (or measure) control for stabilization [41].

The basic problem in studying the solution of MDE (2) comes due to the product $g(\cdot) d u$. When $g(\cdot)$ is a continuous function of time only, so that the discontinuities in $u$ do not cause any discontinuity in $g(\cdot)$, then this product is well-defined. In that case, [36] generalized Caratheodory's concept of solution for systems of form (2) while studying such systems in the context of optimal control. When $g(\cdot)$ is a function of the state, then $g(\cdot)$ becomes discontinuous at the instant when $x(\cdot)$ is potentially discontinuous due to discontinuity in $u(\cdot)$. In that case, the product $g(\cdot) d u$ is not well-defined and an appropriate notion of solution must be defined. Dating back to the work of Kurzweil [21], one approximates the measure $d u(\cdot)$ with a sequence of derivatives of continuously differentiable inputs and the limit of corresponding sequence of solutions is called a solution.

Continuing with this basic idea of defining the solution as a limit of sequences, Sussmann [40] studied such differential equations to develop solution concept for a class of stochastic differential equations using the theory of ordinary differential equations, but only considered singularly continuous inputs. 
Based on his work, $[4,6,12]$ generalized the idea to consider discontinuous inputs, and in general, the functions of bounded variation. From stability point of view, the work of [11] talks about stabilization in terms of existence of an input function that makes some Lyapunov function decrease along the trajectories of the system (2).

A class of systems closely related to system (2) is that of impulsive differential equations (called impulsive ODEs henceforth) which are described by ODEs and a sequence of discrete time instants at which the state is reset according to a predefined reset map. Stability of such systems has been widely studied in the literature, see for example the books [17, 22] that summarize the results on asymptotic stability. To avoid confusion, the notion of stability with respect to two measures considered in [22, 23] has a different meaning: in their work, measure refers to some function of the state (such as the Euclidean norm, or distance from a set) whose convergence is to be studied, whereas in our work the measure refers to the distributional derivative of a function with the bounded variation. The more recent papers $[14,19,25]$ deal with inputto-state stability of impulsive ODEs. For the most part, the stability results for such systems give conditions on the vector field (for continuous dynamics), the jump maps (for discrete dynamics), and the frequency with which the impulses in the system appear.

\subsection{Contribution}

In our work, we will adopt the solution framework proposed in [40] and later developed in $[4,6,12]$. A brief account of this solution methodology is collected in Section 3 and is also used in deriving conditions for stability of the system, which is the main topic of this paper. The two notions of stability for MDEs considered in this article are:

- Asymptotic stability, where we study the problem of convergence of state trajectories to the origin. We propose sufficient conditions in terms of a Lyapunov function associated with the vector fields $f(\cdot)$ and $g(\cdot)$, and identify the class of inputs $u(\cdot)$ which results in asymptotic stability of the system. As a particular case of our results, we can recover some of the results on stability of switched systems, and a certain class of impulsive ODEs. In this regard, our modeling framework allows for (time-dependent) switching vector fields, and more than one kind of state reset map.

- Input-to-state stability (ISS) with respect to variation: The other notion of stability considered in this paper presents a generalization of integral-ISS [3, 39], which is a variant of the more classical notion of ISS with respect to essential supremum norm of the input, introduced in [38]. If $u(\cdot)$ is continuously differentiable (which means $d u=\dot{u} d t$ ), then system $(2)$ is integral-ISS with respect to $\dot{u}(\cdot)$ if

$$
|x(t)| \leq \beta\left(\left|x\left(t_{0}\right)\right|, t-t_{0}\right)+\gamma\left(\int_{t_{0}}^{t}|\dot{u}(s)| d s\right),
$$

where $\beta(\cdot)$ is a class $\mathcal{K} \mathcal{L}$ function, and $\gamma(\cdot)$ is a class $\mathcal{K}_{\infty}$ function (see Section 4 for definitions). Recalling that the integral norm of $\dot{u}(\cdot)$ is nothing but the total variation of the input $u(\cdot)$, the inequality (3) basically determines how the maximum norm of the state trajectories is related to the total variation of the input $u(\cdot)$. If we now allow the inputs to be functions of bounded variation, which are not necessarily continuously differentiable, 
so that $d u$ represents a measure, then our goal is to compute an estimate of the form $(3)$ by replacing ${ }^{1}$ the integral norm of $\dot{u}(\cdot)$ with the total variation of $u(\cdot)$. The difficulties in handling this larger class of inputs arise due to possibly discontinuous state trajectories, and the calculations involving differential measure associated with functions of bounded variation.

Results on asymptotic stability of MDEs have also appeared in $[13,24,33]$. However, the solution concept adopted in these works is different from ours, and in general, their solution is not continuous with respect to inputs and may not even be defined for certain inputs (see Example 1 for details). Because of which, the proposed stability conditions are different in our work. To the best of our knowledge, the notion of ISS with respect to variation for MDEs has not been considered in the literature.

On the other hand, the notion of asymptotic and input-to-state stability has been considered extensively for the class of impulsive ODEs, see for example $[17,19,22]$. In essence, the hypotheses used in the formulation of stability results in our work are different than what we encounter in the literature on impulsive ODEs. To compare the approach of this paper with existing results on stability of impulsive systems, our results do not require the explicit knowledge of the jump map, and they do not place any constraints on the frequency with which the state resets occur. We, however, put constraints on the variation of the input (whose distributional derivative drives the system dynamics) and this is not something we have so far observed in the current literature. The source of this difference is basically due to the modeling framework used in different approaches. Impulsive ODEs typically model piecewise absolutely continuous trajectories where the drift vector field, and the state reset map are fixed. The system description (2) has the advantage of modeling switching vector fields and (different) state reset maps (see Section 4.3 and 4.4); it doesn't rely on the knowledge of when the impulses occur to determine the stability of the system (which makes it robust with respect to perturbations in the impulsive times); it allows for accumulation of jumps in finite time (something which is never considered in impulsive ODEs); and lastly it can model noisy paths (such as Cantor function, or more generally singular continuous functions) which in general are treated using stochastic methods [40].

\subsection{Motivation}

In order to provide the motivation for the class of systems given in (2) and the stability notions considered in this paper, let us introduce an example in the context of optimal control and mention certain practical systems that are driven by measure control.

\subsubsection{Optimal Control Example}

Consider a scalar system described by following differential equation:

$$
\dot{x}=-x+w, \quad x(0)=x_{0} \in \mathbb{R} .
$$

1 It must be noted that for a measure $d u$, the supremum norm cannot be defined and that is why the problem of ISS using essential supremum norm is not of interest for system (2) (also see Section 1.3.1 for an example). One may choose to call the proposed notion of stability as integral-ISS with respect to $d u$, but in standard text books on functional analysis, integral norm of a measure $d u$ is more commonly represented by the total variation of $u(\cdot)$. Hence we choose to work with the more standard terminology of total variation, while keeping in mind that conceptually it represents the integral norm of the input driving the system dynamics. 
For a given $t_{1}>0$, we want to find $w(\cdot)$ such that $x\left(t_{1}\right)=x_{1} \in \mathbb{R}$, and moreover the integral norm of $w(\cdot)$ is minimized. In other words, we consider the following optimal control problem:

$$
\begin{gathered}
\min _{w} \int_{0}^{\infty}|w(s)| d s \\
\text { subject to } \dot{x}=-x+w ; \quad x(0)=x_{0}, \quad \text { and } \quad x\left(t_{1}\right)=x_{1} .
\end{gathered}
$$

By solving the system equation (4), one can see that in order to have $x\left(t_{1}\right)=$ $x_{1}$, we must have

$$
\int_{0}^{t_{1}} e^{s} w(s) d s=e^{t_{1}} x_{1}-x_{0}
$$

Note that $\left|\int_{0}^{t_{1}} e^{s} w(s) d s\right|<e^{t_{1}} \int_{0}^{t_{1}}|w(s)| d s$, so for any control that drives the state to desired terminal state, we must have $\int_{0}^{t_{1}}|w(s)| d s>\left|x_{1}-e^{-t_{1}} x_{0}\right|$. For a generic set of points $x_{0}, x_{1} \in \mathbb{R}$, which satisfy $x_{1} \neq e^{-t_{1}} x_{0}$, it is not possible for any function $w(\cdot)$ to drive the state $x(\cdot)$ to the desired terminal point and has an integral norm no greater than $\left|x_{1}-e^{-t_{1}} x_{0}\right|$. In fact, this optimal control problem doesn't have a solution over the space of Lebesgue-measurable functions. However, if we relax the search space to include distributions, or measures, and let $\delta_{[0]}$ denote the Dirac impulse concentrated at $t=0$, then it is seen that the generalized function $w^{*}=\left(x_{1}-x_{0} e^{-t_{1}}\right) \cdot \delta_{\left[t-t_{1}\right]}$ achieves the desired objective with minimum integral norm. Such examples provide the motivation of studying systems with distributional inputs.

To motivate the stability notions addressed in this paper, let us first consider a sequence of functions $\left\{w_{k}\right\}_{k=1}^{\infty}$ which approximates ${ }^{2} w^{*}$ in appropriate sense. For instance, we may take the sequence of piecewise constant functions:

$$
w_{k}(t):= \begin{cases}k\left(x_{1}-x_{0} e^{-t_{1}}\right), & t_{1} \leq t<t_{1}+\frac{1}{k} \\ 0, & \text { otherwise }\end{cases}
$$

for $k \in \mathbb{N}$, and $t \geq 0$. Let $x_{k}(\cdot)$ and $x^{*}(\cdot)$ denote the trajectories of (4) corresponding to the inputs $w=w_{k}(\cdot)$, and $w=w^{*}$, respectively. When a system is subjected to inputs which have very large $\mathcal{L}_{\infty}$-norm, but relatively small $\mathcal{L}_{1}$-norm, then it is useful to compute the estimates on the growth of the state trajectories in terms of the $\mathcal{L}_{1}$-norm of the inputs. In this particular example, it is easily seen that, for large values of $k \in \mathbb{N}$, while the $\mathcal{L}_{\infty}$-norm of $w_{k}(\cdot)$ doesn't provide any useful estimates for $\left\|x_{k}\right\|_{\infty}$, the use of $\mathcal{L}_{1}$-norm gives the following inequality:

$$
\left|x_{k}(t)\right| \leq e^{-t}\left|x_{0}\right|+\int_{0}^{t}\left|w_{k}(s)\right| d s, \quad t \geq 0
$$

which, using the semigroup property, leads to the following estimate:

$$
\left|x_{k}(t)\right| \leq e^{-t}\left|x_{0}\right|+e^{-\left(t-\bar{t}_{1}\right)} \int_{0}^{\bar{t}_{1}}\left|w_{k}(s)\right| d s, \quad t>\bar{t}_{1}:=t_{1}+1 .
$$

\footnotetext{
2 The sequence $\left\{w_{k}\right\}_{k=1}^{\infty}$ converges to $w^{*}$ in the distributional sense only, which means that

$$
\lim _{k \rightarrow \infty} \int_{0}^{\infty} \phi(s) w_{k}(s) d s=\int_{0}^{\infty} \phi(s) w^{*}(s) d s
$$

for every continuous function $\phi(\cdot)$ supported over a compact domain. This notion of convergence is also called weak ${ }^{*}$-convergence.
} 
Since the right-hand is uniformly bounded for each $k \in \mathbb{N}$, we can compute meaningful estimates of $\left\|x_{k}\right\|_{\infty}$ this way. The sequence of functions $x_{k}(\cdot)$ converges pointwise to $x^{*}(\cdot)$ (but not uniformly), so these estimates are also useful for the actual trajectory (which results from the measure control). In general, we are interested in systems subjected to the distributional inputs (which are bounded in the integral sense only), and in order to characterize the growth of state trajectories for such systems, it would be cumbersome to work every time with a sequence of functions that approximate the measure control, and check that the resulting trajectories converge pointwise to the desired trajectory, in order for the estimates obtained from ODE approximations to be meaningful. For that reason, we want to obtain the norm estimates of the function $x^{*}(\cdot)$ directly in terms of $w^{*}$. Also, by using the triangle inequality $\left|x_{k}(t)-x^{*}(t)\right| \leq\left|x_{k}(t)\right|+\left|x^{*}(t)\right|$, we can get an upper bound on how much the trajectories $x_{k}(\cdot)$ (obtained by the approximations of the measure control) differ from the optimal trajectory $x^{*}(\cdot)$, albeit conservative.

In the approach adopted in this paper, we introduce a function $u^{*}(\cdot)$, which for this example could be taken as:

$$
u^{*}(t)= \begin{cases}C, & 0 \leq t<t_{1} \\ C\left(x_{1}-x_{0} e^{-t_{1}}\right), & t \geq t_{1}\end{cases}
$$

with some constant $C>0$. Then $w^{*}$ is the distributional derivative of $u^{*}(\cdot)$, and we use the notation $w^{*}=d u^{*}$ to write system (4) in the form of system (2). One can now use the total variation of the function $u^{*}(\cdot)$ to denote the integral norm of $w^{*}$, which is in turn used to bound the maximum norm of $x^{*}(\cdot)$. This way, we can also determine whether the jumps in state trajectories are related to the jumps in the inputs through a finite gain. The notion of stability considered in Section 5 is exactly aimed at answering such questions and provides the estimates on the $\mathcal{L}_{\infty}$-norm of the state trajectories in terms of the total variation of the input function. It will turn out (see Theorem 2) that the system considered in this example is ISS with respect to the variation of the input $u(\cdot)$.

\subsubsection{Practical Systems with Impulsive Control}

From practical standpoint, such optimal control problems (which require measure controls) also arise in designing control algorithms that would derive a spacecraft from one point to another using the minimum amount of fuel with no constraints on the vehicle's thrust vector, see [31] for mathematical formulation and further details. It turns out that in order to compute the optimal solution using the variational equations associated with the optimal control problem, the problem reduces to finding a control with minimum variation that achieves the desired endpoint configuration. The solution to such problem exists only in the space of functions with bounded variation and the expression for optimal thrust vector involves the derivatives of such functions, so that the resulting system is of the form (2). Such mathematical formalisms for satellite control are still being studied $[9,10,35]$ and provide a good motivation to study systems with measure controls.

In these examples, we have seen that the framework of MDEs is particularly useful when a very fast response is desired from the system. Among other utilities, there are models in advertising management [16], population control in ecosystem [43, 44], economics and quantum electronics [15] that use the formalism measure controls in their description. Our results on ISS with 
respect to variation could be used to study robustness with respect to small errors in such impulsive inputs. Another advantage of considering MDEs is that we can possibly model Zeno trajectories because $u(\cdot)$ as a function of bounded variation may exhibit infinitely many discontinuities in a finite interval. This also makes the simulation of Zeno trajectories possible as one no longer uses the classical event-based schemes to model discontinuities but rather a time-stepping algorithm is employed as demonstrated in [1]. Other than the discontinuities, singularly continuous inputs [40] (such as a random process with continuous sample path) may also be considered.

\section{Preliminaries}

We now recall some preliminary results related to functions of bounded variation which will be used later on. For a function $u:\left[t_{0}, T\right] \rightarrow \mathbb{R}^{m}$, the total variation is a non-decreasing function and is defined for $t \in\left[t_{0}, T\right]$ as $\operatorname{var}_{u}\left(t_{0} ; t\right)=\sup \sum_{i=1}^{k}\left|u\left(\tau_{i}\right)-u\left(\tau_{i-1}\right)\right|$, where the supremum is taken over all integers $k$, and all possible choices of the sequence $\left\{\tau_{i}\right\}$ such that $t_{0}=\tau_{0}<\tau_{1}<\cdots<\tau_{k}=t$. The function $u(\cdot)$ is said to be of bounded variation $(B V)$ on $\left[t_{0}, T\right]$ if $\operatorname{var}_{u}\left(t_{0} ; T\right)<\infty$. If it is right continuous with bounded variation we denote it as $R C B V$. It is locally $R C B V$ if this holds for any compact interval $\left[t_{0}, T\right] \subset \mathbb{R}$. If $u(\cdot)$ is $B V$ on $\left[t_{0}, T\right]$ then it has at most a countable number of jump discontinuities. Moreover, it has right and left limits everywhere. The right and left limits of a function at $t \in\left[t_{0}, T\right]$ are denoted as $u\left(t^{+}\right):=\lim _{s \searrow t} u(s)$ and $u\left(t^{-}\right):=\lim _{s \nearrow t} f(s)$, respectively, and are well defined for $B V$ functions. In this notation, right continuity of $u(\cdot)$ in $t$, means that $u\left(t^{+}\right)=u(t)$.

For an interval $I \subseteq \mathbb{R}$, we denote by $\mathcal{L}_{1}\left(I, \mathbb{R}^{n} ; \nu\right)$ and $\mathcal{L}_{1}^{\text {loc }}\left(I, \mathbb{R}^{n} ; \nu\right)$ the space of integrable and locally integrable functions, respectively, from $I$ to $\mathbb{R}^{n}$ with respect to the measure $\nu$. If the measure is not specified then the integration is with respect to the Lebesgue measure. Similarly, $\mathcal{C}^{1}\left(\mathbb{R}, \mathbb{R}^{n}\right)$ denotes the set of continuously differentiable functions from $\mathbb{R}$ to $\mathbb{R}^{n}$.

\subsection{System class}

The class of systems studied in this paper is represented by:

$$
d x=f(x) d t+G(x) d u
$$

where $G(x): \mathbb{R}^{n} \rightarrow \mathbb{R}^{n \times m}$ is a matrix whose $j$-th column is denoted by $g_{j}(x), j=1, \ldots, m$, and the input $u:[0, \infty) \rightarrow \mathbb{R}^{m}$ is assumed to be a rightcontinuous function with locally bounded variation. It will be assumed that the entries of the vector fields $f(\cdot), g_{1}(\cdot), \ldots, g_{m}(\cdot)$ are continuously differentiable.

When dealing with systems of the form $(5)$, if $u(\cdot)$ is discontinuous, then $d u$ contains a Dirac impulse, and $x(\cdot)$ is possibly discontinuous. In that case, a problem arises with the interpretation of the product $G(x) d u$ since the product of a discontinuous function and a Dirac impulse is not well-defined in general. How one interprets this product, basically determines the notion of solution also; see Section 3 for various notions of solutions adopted for system (5) in the literature. In our work, we consider $x(\cdot)$ to be the solution if it varies continuously with respect to the inputs. 


\subsection{Measure-theoretic notions}

For a non-decreasing, right-continuous function $u(\cdot):\left[t_{0}, T\right] \rightarrow \mathbb{R}^{m}$ of bounded variation, we can associate a vector-valued Lebesgue-Stieltjes measure $\mu$ such that

$$
\mu((s, t])=u(t)-u(s), \quad \forall(s, t] \subseteq\left[t_{0}, T\right]
$$

If $u(\cdot)$ possess a discontinuity at some time instant $t_{i} \in(s, t]$, then we say that $\left\{t_{i}\right\}$ is an atom of the measure $\mu$ and $\mu\left(\left\{t_{i}\right\}\right)=u\left(t_{i}^{+}\right)-u\left(t_{i}^{-}\right)$. If each component of $\mu$ is denoted by $\mu_{j}, j=1, \ldots, m$, then the total variation of $\mu$ is given by $|\mu|:=\sum_{j=1}^{m} \mu_{j}$. It is well-known that every $R C B V$ function could be written as a difference of two right-continuous non-decreasing functions, that is, there exist some functions $u^{+}(\cdot)$ and $u^{-}(\cdot)$ (both non-decreasing in each of its component) such that $u=u^{+}-u^{-}$. We can then associate the Lebesgue-Stieltjes measure with each of these functions and denote them by $\mu^{+}$and $\mu^{-}$, respectively. Thus, if $\mu$ denotes the Lebesgue-Stieltjes measure associated with an $R C B V$ function (not necessarily nondecreasing) $u(\cdot)$, then $\mu=\mu^{+}-\mu^{-}$. The total variation of this measure is denoted by $|\mu|$, and is equal to $\left|\mu^{+}\right|+\left|\mu^{-}\right|$. We will use the notation $|\mu|_{\left[t_{0}, t\right]}$ to denote the total variation of the measure $\mu$ over the interval $\left[t_{0}, t\right]$ and it is seen that $|\mu|_{\left[t_{0}, t\right]}=\operatorname{var}_{u}\left(t_{0} ; t\right)$.

The distributional derivative $d u$ (also called the differential measure [2, $28]$ ) introduced in (5) could be seen as a generalized notion of derivative and satisfies the following relation:

$$
\mu((s, t])=u(t)-u(s)=\int_{(s, t]} d u .
$$

One may verify that, for absolutely continuous functions, if we let $d u=\dot{u} d t$ with $\dot{u}(\cdot)$ a Lebesgue-measurable function denoting the classical derivative of $u(\cdot)$, then the above relation is obtained simply from the fundamental theorem of calculus. It is noted that the function $u(\cdot)$ is decreasing (or, non-decreasing) over an interval if $d u<0$ (resp., $d u \leq 0$ ) over that entire interval.

We also recall that the function $u(\cdot)$, being locally $R C B V$, has countably many discontinuities over the interval $\left[t_{0}, \infty\right)$, and we let $\mathcal{I}$ denote a countable set that indexes the discontinuities of $u(\cdot)$. The measure $\mu$ associated with $u(\cdot)$ admits the following decomposition: $\mu=\mu_{\mathrm{ac}}+\mu_{\text {sing }}+\mu_{d}$, where $\mu_{\mathrm{ac}}$ is absolutely continuous with respect to Lebesgue measure, $\mu_{\text {sing }}$ is associated with a singular function which is continuous everywhere and differentiable Lebesgue-almost everywhere with zero derivative (e.g., the Cantor function). We use the notation $\mu_{c}:=\mu_{\mathrm{ac}}+\mu_{\text {sing }}$ to denote the continuous part of $\mu$. The measure of the discontinuous part is given by $\mu_{d}:=\sum_{i \in \mathcal{I}} \mu\left(\left\{t_{i}\right\}\right)$, where $\mu\left(\left\{t_{i}\right\}\right)$ denotes the variation at time $t_{i}$ where $u(\cdot)$ has jump discontinuity. To clarify some notation later and better understand the definition of the measure $\mu$, note that if $u(\cdot)$ is $R C B V$ on an interval $[s, t]$ with a single discontinuity at $t_{i}, s<t_{i}<t$, then $\mu_{d}([s, t])=\mu\left(\left\{t_{i}\right\}\right)=u\left(t_{i}^{+}\right)-u\left(t_{i}^{-}\right)$, and $\mu_{c}([s, t])=$ $\mu([s, t])-\mu_{d}([s, t])=u(t)-u\left(s^{-}\right)-\mu_{d}([s, t])$.

\section{Solution Concepts}

As stated in the introduction, the solution of system (5) is well-defined for an absolutely continuous input $u(\cdot)$. However, in this paper, it is assumed that the function $u(\cdot)$ is only locally $R C B V$ in which case the term $d u$ denotes the differential measure associated with $u(\cdot)$. When $g(\cdot)$ is assumed to be a 
continuous function of time only, and not of state, there is no ambiguity in the interpretation of the solution. In that case, Carathédory's notion of solution for ordinary differential equation has been extended to arrive at similar conditions for existence and uniqueness of solution to system (5) in the class of locally $R C B V$ functions, see [36]. However, with the vector field $g(\cdot)$ considered as a function of the state variable, the notion of solution is not so straight forward. The following example illustrates some degree of difficulty in obtaining the solution to MDE (5).

Example 1 Consider the scalar system (5) with $f(x)=0, G(x)=x$, and $u(t)=c_{1} H(t)$, where $c_{1}>0$, and $H(t)=\left\{\begin{array}{ll}0, & t_{0}<t<t_{1} \\ 1, & t_{1} \leq t<\infty\end{array}\right.$. This gives $d u=c_{1} \delta_{\left[t-t_{1}\right]}$, where $\delta_{[0]}$ denotes the Dirac measure at time $t=0$. It is clear that $x(t)=x\left(t_{0}\right)$, for $t_{0} \leq t<t_{1}$. For $t \geq t_{1}$, if we pick $x(t)=\left(1+c_{1}\right) x\left(t_{0}\right)$ then the equation $\int_{\left\{t_{1}\right\}} d x=x\left(t_{1}^{-}\right) \int_{\left\{t_{1}\right\}} d \bar{u}$ holds; however, the solution $x(t)=$ $x\left(t_{0}\right) /\left(1-c_{1}\right)$ satisfies $\int_{\left\{t_{1}\right\}} d x=x\left(t_{1}^{+}\right) \int_{\left\{t_{1}\right\}} d u$. Another solution is obtained by approximating $u(\cdot)$ with a sequence $\left\{u_{k}\right\}_{k=1}^{\infty}$ of continuously differentiable functions. Then for each element of the sequence, the resulting solution is obtained by solving $\frac{\dot{x}_{k}}{x}(t)=\dot{u}_{k}(t)$, which leads to $x_{k}(t)=x_{k}\left(t_{0}\right) e^{u_{k}(t)}$. One then takes $x(\cdot)$ to be the limit of the sequence $\left\{x_{k}\right\}_{k=1}^{\infty}$ and let $x(t)=x\left(t_{0}\right) e^{u(t)}$ to be the solution. In terms of the original system description in differential form, this last solution satisfies $\int_{\left\{t_{1}\right\}} d x=\tilde{x} \int_{\left\{t_{1}\right\}} d u$, for some $\tilde{x} \in\left[x\left(t_{1}^{-}\right), x\left(t_{1}^{+}\right)\right]$. To prove the last claim, we introduce the function $\Phi(v)=e^{v} x_{0}$, for $v \in\left[0, c_{1}\right]$. Then by the mean value theorem, there exists $\tilde{v} \in\left[0, c_{1}\right]$ such that $\left.\frac{\partial \Phi}{\partial v}\right|_{v=\tilde{v}}=$ $\frac{\Phi\left(c_{1}\right)-\Phi(0)}{c_{1}}$, that is, $e^{\tilde{v}} x_{0}=\frac{e^{c_{1}} x_{0}-x_{0}}{c_{1}}$ and we note that $\int_{\left\{t_{1}\right\}} d x=e^{c_{1}} x_{0}-x_{0}$, $\int_{\left\{t_{1}\right\}} d u=c_{1}$, and $\tilde{x}:=e^{\tilde{v}} x_{0} \in\left[x\left(t_{1}^{-}\right), x\left(t_{1}^{+}\right)\right]$. This last argument indicates that at the points of discontinuity of $x(\cdot)$ at $t_{i}$, we have $\frac{d x}{d u}\left(t_{i}\right)=g(\tilde{x})$, where $\tilde{x}$ is some point on the solution curve of the ODE $\dot{z}=\left(u\left(t_{i}^{+}\right)-u\left(t_{i}^{-}\right)\right) g(z)$ solved over the interval $[0,1]$ with initial condition $z(0)=x\left(t_{i}^{-}\right)$.

It is seen that the first two notions of solutions considered in the example are not continuous with respect to the inputs. In general, such solutions won't be unique either [18]. However, the last solution obtained by approximating $u(\cdot)$ with continuously differentiable functions has some nice properties and we will develop results on stability with respect to such solutions.

In order to develop an appropriate notion of solution for system (5), we recall the work of $[4,12,40]$. Towards that end, let $\Phi_{G}\left(s ; z_{0}, v\right)$ denote the Carathéodory solution of the ordinary differential equation

$$
\dot{z}(t)=\sum_{j=1}^{m} v_{j} g_{j}(z(t)), \quad z(0)=z_{0}
$$

at time $t=s$ with initial condition $x_{0}$, i.e., $\Phi_{G}\left(0 ; z_{0}, v\right)=z_{0}$. It also follows that $\Phi_{G}\left(s ; z_{0}, 0\right)=z_{0}$ for each $s \in \mathbb{R}$. Now let ${ }^{3}$

$$
h_{j}(z, v):=\int_{0}^{1} g_{j}\left(\Phi_{G}(s ; z, v)\right) d s
$$

and the Cauchy problem that we consider is

$$
d x=f(x(t)) d t+\sum_{j=1}^{m} h_{j}\left(x\left(t^{-}\right), u\left(t^{+}\right)-u\left(t^{-}\right)\right) d u .
$$

\footnotetext{
3 The choice of the interval $[0,1]$ is arbitrary and could be replaced by any other compact interval without changing the development much.
} 
It is seen that the solution of (6) coincides with the solutions of (5) whenever $u(\cdot)$ is continuous, since in that case $u\left(t^{+}\right)=u\left(t^{-}\right)$for all $t$, and $h_{j}(x, 0)=$ $g_{j}(x)$. Formally, the solution to system (5) is defined as follows:

Definition 1 For a given locally $R C B V$ input $u:\left[t_{0}, \infty\right) \rightarrow \mathbb{R}^{m}$, a locally $R C B V$ function $x:\left[t_{0}, \infty\right) \rightarrow \mathbb{R}^{n}$ is called a solution of (5) if it satisfies the following:

$$
\int_{\mathcal{B}} d x=\int_{\mathcal{B}} f(x(t)) d t+\sum_{j=1}^{m} \int_{\mathcal{B}} h_{j}\left(x\left(t^{-}\right), u\left(t^{+}\right)-u\left(t^{-}\right)\right) d u
$$

for every Borel measurable set $\mathcal{B} \subset\left[t_{0}, \infty\right)$.

To study the existence and uniqueness of the Cauchy problem with inputs of bounded variation (6), the basic idea is to introduce a graph completion of the input $u(\cdot)$ which are Lipschitz continuous. An auxiliary system is then introduced which is driven by this graph completion, and the solution of this system can be studied in the classical sense. Once the existence of solution is verified in the new coordinates, the solutions of the auxiliary system are mapped back into the original coordinates. Some details of this development are given in Appendix A, and for further details, we refer the reader to [12].

We study equation (6) not only because it generalizes the solution of (5), but also because its solution varies continuously with respect to the input. In particular, we have the following result:

Proposition 1 ([12, Theorem 4.2]) Let $u:\left[t_{0}, \infty\right) \rightarrow \mathbb{R}^{m}$ be a locally $R C B V$ function and consider a sequence $u_{k}:\left[t_{0}, \infty\right) \rightarrow \mathbb{R}^{m}$ of locally $R C B V$ functions. Assume that, for every compact interval $I \subset\left[t_{0}, \infty\right)$,

- it holds that $\lim _{k \rightarrow \infty} u_{k}(t)=u(t)$ for almost every $t \in I$, and

- and $\lim _{k \rightarrow \infty} \operatorname{var}_{u_{k}}\left(t_{0} ; t\right)=\operatorname{var}_{u}\left(t_{0} ; t\right)$.

Let $x_{k}(\cdot), x(\cdot)$ be locally $R C B V$ functions obtained as solutions to (6) corresponding to $u_{k}(\cdot)$, and $u(\cdot)$, respectively. Then

$$
\lim _{k \rightarrow \infty} x_{k}(t)=x(t)
$$

for each $t \in I \subset\left[t_{0}, \infty\right)$, where $u(\cdot)$ is continuous.

From the system description in $(6)$, the solution $x(\cdot)$ at the discontinuities is characterized as follows:

Proposition 2 (Jump Characterization) At the atoms $\left\{t_{i}\right\}$ of $\mu$, we have

$$
x\left(t_{i}^{+}\right)=\Phi_{G}\left(1 ; x\left(t_{i}^{-}\right), u\left(t_{i}^{+}\right)-u\left(t_{i}^{-}\right)\right) .
$$

To see how we arrive at the formula for $x\left(t_{i}^{+}\right)$, it follows from the definition of the functions $h_{j}(\cdot, \cdot)$ that

$$
\begin{aligned}
\sum_{j=1}^{m} v_{j} h_{j}(z, v) & =\int_{0}^{1} \sum_{j=1}^{m} v_{j} g_{j}\left(\Phi_{G}(s ; z, v)\right) d s \\
& =\int_{0}^{1} \frac{\partial \Phi_{G}}{\partial s}(s ; z, v) d s \\
& =\Phi_{G}(1 ; z, v)-\Phi_{G}(0 ; z, v) \\
& =\Phi_{G}(1 ; z, v)-z .
\end{aligned}
$$


If $u(\cdot)$ is discontinuous at $t_{i}$, then $(6)$ is interpreted as follows:

$$
x\left(t_{i}^{+}\right)-x\left(t_{i}^{-}\right)=\sum_{j=1}^{m} h_{j}\left(x\left(t_{i}^{-}\right), u\left(t_{i}^{+}\right)-u\left(t_{i}^{-}\right)\right)\left(u_{j}\left(t_{i}^{+}\right)-u_{j}\left(t_{i}^{-}\right)\right)
$$

and from the above calculations it follows that

$$
x\left(t_{i}^{+}\right)=\Phi_{G}\left(1 ; x\left(t_{i}^{-}\right), u\left(t_{i}^{+}\right)-u\left(t_{i}^{-}\right)\right) .
$$

One can also verify that the solution notion proposed in Example 1 is indeed consistent with the result given in Proposition 2.

Remark 1 There are several other results in the literature on continuity of solutions similar to Proposition 1 under various hypotheses on the control vector fields. The basic objective is to study the solution of (5) as the limit of solutions obtained from differentially regular inputs. In case, the control vector fields are commutative [6], or they form a solvable Lie algebra [7], one obtains this continuity of solutions with respect to regular controls without imposing any bounds on the total variation as done in Proposition 1 . The bound on the total variation guarantees that we have convergence in the sense of graph completions, which in turn guarantees that the corresponding classical solutions converge (see Appendix A for details on solutions obtained from graph completions).

\section{Asymptotic Stability}

We now study the stability properties of system (5) with respect to the origin. Two variants of the stability notions will be considered. The first one relates to the attractivity of the state trajectories towards the origin for a specific class of inputs and is studied in Section 4.1. Then, in Section 5, we derive weaker conditions to establish the boundedness of the state trajectories in terms of the total variation of the input.

Before stating the main result, we recall some standard definitions: A function $\alpha:[0, \infty) \rightarrow[0, \infty)$ belongs to class $\mathcal{K}$ if it is continuous, strictly increasing, and $\alpha(0)=0$. If, in addition, $\alpha(\cdot)$ is unbounded then $\alpha(\cdot)$ belongs to class $\mathcal{K}_{\infty}$. A function $\beta:[0, \infty) \times[0, \infty) \rightarrow[0, \infty)$ belongs to class $\mathcal{K} \mathcal{L}$ if $\beta(\cdot, t) \in \mathcal{K}$ for each $t \geq 0, \beta(r, \cdot)$ is strictly decreasing for each $r \geq 0$ and $\beta(r, t) \rightarrow 0$ as $t \rightarrow \infty$.

\subsection{Sufficient Condition}

In this section, the following notion of stability is considered.

Definition 2 We call the system (5) uniformly asymptotically stable over a set of inputs $\mathcal{U}$ if there exists a class $\mathcal{K} \mathcal{L}$ function $\beta(\cdot, \cdot)$ such that every state trajectory $x(\cdot)$ resulting from the input $u \in \mathcal{U}$ as a solution of (5) satisfies $|x(t)| \leq \beta\left(\left|x\left(t_{0}\right)\right|, t-t_{0}\right)$.

We now state our first main result.

Theorem 1 (Asymptotic Stability) Assume that there exist a continuously differentiable function $V: \mathbb{R}^{n} \rightarrow \mathbb{R}_{+}$, some class $\mathcal{K}_{\infty}$ functions $\alpha_{1}(\cdot)$, 
$\alpha_{2}(\cdot)$, and some constants $a, \underline{b}, \bar{b} \in \mathbb{R}$ such that the following holds for each $x \in \mathbb{R}^{n}$ :

$$
\begin{aligned}
& \alpha_{1}(|x|) \leq V(x) \leq \alpha_{2}(|x|), \\
& \langle\nabla V(x), f(x)\rangle \leq a V(x), \\
& \underline{b}_{j} V(x) \leq\left\langle\nabla V(x), g_{j}(x)\right\rangle \leq \bar{b}_{j} V(x), \quad j=1, \ldots, m .
\end{aligned}
$$

Let $\mathcal{U}_{c, \theta}$ denote the class of inputs satisfying:

$$
a\left(t-t_{0}\right)+\sum_{j=1}^{m} \bar{b}_{j} \mu_{j}^{+}\left(\left[t_{0}, t\right]\right)-\sum_{j=1}^{m} \underline{b}_{j} \mu_{j}^{-}\left(\left[t_{0}, t\right]\right) \leq c-\theta\left(t-t_{0}\right)
$$

for some $c \in \mathbb{R}$ and $\theta \in \mathcal{K}_{\infty}$. Then, system (5) is uniformly asymptotically stable over $\mathcal{U}_{c, \theta}$.

Proof Consider the candidate Lyapunov function $V(\cdot)$ satisfying the hypotheses in the statement of the theorem. Since $V(\cdot)$ is continuously differentiable and the solution $x(\cdot)$ is locally $R C B V$, the composition function $W: \mathbb{R} \rightarrow \mathbb{R}_{+}$ defined as $t \mapsto V(x(t))$ is also locally $R C B V$ [29, Theorem 3]. Over an interval $\left(t_{i}, t_{i+1}\right)$ between the two discontinuities of the input $u(\cdot)$, the state trajectory $x(\cdot)$ is continuous and the differential measure of $W(\cdot)$ is computed by

$$
d W=\langle\nabla V(x), f(x)\rangle d t+\sum_{j=1}^{m}\left\langle\nabla V(x), g_{j}(x)\right\rangle d u_{j}
$$

Writing $u=u^{+}-u^{-}$and using (7b), (7c) along with $d u_{j}^{+} \geq 0$ and $d u_{j}^{-} \geq 0$ for each $j=1, \ldots, m$, we get

$$
d W \leq a W d t+\sum_{j=1}^{m} \bar{b}_{j} W d u_{j}^{+}-\sum_{j=1}^{m} \underline{b}_{j} W d u_{j}^{-} .
$$

For $t \in\left(t_{i}, t_{i+1}\right)$, let $\rho_{i}(t):=a\left(t-t_{i}\right)+\sum_{j=1}^{m} \bar{b}_{j} \mu_{j}^{+}\left(\left(t_{i}, t\right)\right)-\sum_{j=1}^{m} \underline{b}_{j} \mu_{j}^{-}\left(\left(t_{i}, t\right)\right)$, and

$$
W_{c, i}(t):=e^{-\rho_{i}(t)} W(t) .
$$

The use of product rule and chain rule for differential of bounded variation functions (recalled from [27, Page 8] and [29, Theorem 3]) gives:

$$
d W_{c, i}=W e^{-\rho_{i}(t)}\left(-a d t-\sum_{j=1}^{m} \bar{b}_{j} d u_{j}^{+}+\sum_{j=1}^{m} \underline{b}_{j} d u_{j}^{-}\right)+e^{-\rho_{i}(t)} d W \leq 0 .
$$

Thus, $W_{c, i}(\cdot)$ is non-increasing on the interval $\left(t_{i}, t_{i+1}\right)$ for each $i \in \mathcal{I}$. Since $W_{c, i}(\cdot)$ is right-continuous and $\rho_{i}\left(t_{i}\right)=0$, we have $W_{c, i}(t) \leq W_{c, i}\left(t_{i}\right)$, which implies that

$$
W(t) \leq e^{\rho(t)} W\left(t_{i}\right)
$$

If $\left\{t_{i}\right\}, i \in \mathcal{I}$, is an atom of the measure $\mu$, then from Proposition $2, x\left(t_{i}^{+}\right)=$ $z_{i}(1)$, where $z_{i}(\cdot)$ satisfies the following ODE:

$$
\dot{z}_{i}(s)=\sum_{j=1}^{m}\left(u_{j}\left(t_{i}^{+}\right)-u_{j}\left(t_{i}^{-}\right) g_{j}\left(z_{i}(s)\right), \quad z_{i}(0)=x\left(t_{i}^{-}\right) .\right.
$$


Under the given hypothesis, it follows that

$$
\begin{aligned}
\dot{V}(z(s)) & \leq \sum_{j=1}^{m}\left\langle\nabla V\left(z_{i}\right),\left(u_{j}\left(t_{i}^{+}\right)-u_{j}\left(t_{i}^{-}\right) g_{j}(z(s))\right\rangle\right. \\
& \leq \sum_{j=1}^{m}\left(\bar{b}_{j} \mu_{j}^{+}\left(\left\{t_{i}\right\}\right)-\underline{b}_{j} \mu_{j}^{-}\left(\left\{t_{i}\right\}\right)\right) V(z(s)) .
\end{aligned}
$$

Using the comparison lemma for ODEs [20, Lemma 3.4], and recalling that $\mu\left(\left\{t_{i}\right\}\right)=u\left(t_{i}^{+}\right)-u\left(t_{i}^{-}\right)$we get

$$
V(z(1)) \leq e^{\sum_{j=1}^{m} \bar{b}_{j} \mu_{j}^{+}\left(\left\{t_{i}\right\}\right)-\underline{b}_{j} \mu_{j}^{-}\left(\left\{t_{i}\right\}\right)} V(z(0)) .
$$

It is noted that $W\left(t_{i}^{-}\right)=V\left(x\left(t_{i}^{-}\right)\right)=V(z(0))$, and $W\left(t_{i}^{+}\right)=V\left(x\left(t_{i}^{+}\right)\right)=$ $V\left(z_{i}(1)\right)$, so that

$$
W\left(t_{i}^{+}\right) \leq e^{\sum_{j=1}^{m} \bar{b}_{j} \mu_{j}^{+}\left(\left\{t_{i}\right\}\right)-\underline{b}_{j} \mu_{j}^{-}\left(\left\{t_{i}\right\}\right)} W\left(t_{i}^{-}\right) .
$$

Combining (9) with (11), and using the countable additivity of the measure $\mu(\cdot)$, we have

$$
\begin{aligned}
W(t) & \leq e^{a\left(t-t_{0}\right)+\sum_{j=1}^{m} \bar{b}_{j} \mu_{j}^{+}\left(\left[t_{0}, t\right]\right)-\underline{b}_{j} \mu_{j}^{-}\left(\left[t_{0}, t\right]\right)} W\left(t_{0}\right) \\
& \leq e^{c-\theta\left(t-t_{0}\right)} W\left(t_{0}\right)
\end{aligned}
$$

where we used (8) to obtain the last inequality. Using (7a) and letting $\beta(r, t):=$ $\alpha_{1}^{-1}\left(e^{c} \alpha_{2}(r)\right) e^{-\theta(t)}$, we obtain

$$
|x(t)| \leq \beta\left(\left|x\left(t_{0}\right)\right|, t-t_{0}\right),
$$

which proves the desired result since $\beta \in \mathcal{K} \mathcal{L}$.

Remark 2 Roughly speaking, the solution of system (5) could be seen as flows along the vector fields $f(\cdot)$ and $g_{j}(\cdot)$. Condition (8) in Theorem 1 basically assigns the weight on how long each of these vector fields should be active for the system to be asymptotically stable. For that, neither $f(\cdot)$, nor any of the control vector fields $g_{j}(\cdot)$ need to be stable in the classical sense. However, appropriate choice of $u(\cdot)$ may render the system asymptotically stable depending on the signs of the scalars $a, \underline{b}_{j}, \bar{b}_{j}$ appearing in (8).

Example 2 Consider a bilinear system with single input

$$
d x=A x d t+B x d u
$$

with the matrix $A$ assumed to be Hurwitz. There exists a symmetric positive definite matrix $P>0$ such that $A^{\top} P+P A=-a P$ for some $a>0$. With $V=x^{\top} P x$, this gives $\langle\nabla V(x), A x\rangle \leq-a V(x)$ for all $x \in \mathbb{R}^{n}$. Moreover, we have $\underline{b} V(x) \leq\langle\nabla V(x), B x\rangle \leq \bar{b} V(x)$ for $\underline{b}=\frac{\lambda_{\min }(\tilde{B})}{\lambda_{\max }(P)}, \bar{b}=\frac{\lambda_{\max }(\tilde{B})}{\lambda_{\min }(P)}$, where $\tilde{B}=P B+B^{\top} P$, and $\lambda_{\max }(\cdot), \lambda_{\min }(\cdot)$ denote the maximum and minimum eigenvalues of a matrix, respectively. If $\lambda_{\min }(\tilde{B})>0$ and $\mathcal{U}_{e d}$ comprises of inputs that are eventually non-increasing ${ }^{4}$ then system (12) is asymptotically stable over $\mathcal{U}_{e d}$ because, for each $u(\cdot) \in \mathcal{U}_{\text {ed }}$, the term $\mu^{+}\left(\left[t_{0}, t\right]\right)$ becomes constant after some large $t$ so that (8) is satisfied for some $c>0$ and $\theta \in \mathcal{K}_{\infty}$. Similarly, if $\lambda_{\max }(\tilde{B})<0$ and $\mathcal{U}_{e i}$ comprises of inputs that are eventually non-decreasing then system (12) is asymptotically stable over $\mathcal{U}_{e i}$ because $\mu^{-}\left(\left[t_{0}, t\right]\right)$ becomes constant after some $t$ large enough, for each $u(\cdot) \in \mathcal{U}_{e i}$.

\footnotetext{
4 A function $h: \mathbb{R} \rightarrow \mathbb{R}$ is called eventually non-increasing if there exists $T$ such that for every $\varepsilon_{2}>\varepsilon_{1} \geq 0$, it holds that $h\left(T+\varepsilon_{2}\right) \leq h\left(T+\varepsilon_{1}\right)$. Similarly, $h(\cdot)$ is called eventually non-decreasing if $-h(\cdot)$ is eventually non-increasing.
} 
As corollaries to Theorem 1, we now discuss several special cases which illustrate the utility of the stability criteria (8) and how it compares to some of the existing results in the literature.

\subsection{Inputs with bounded variation}

We now address a special case of Theorem 1 for a specific class of inputs. Let $\mathcal{U}_{M}$ denote the class of inputs for which $|\mu|_{\left[t_{0}, \infty\right)} \leq M$, that is, the total variation of the inputs is bounded by some constant $M>0$. For the case when $a<0$, the following corollary shows that $\mathcal{U}_{M} \subset \mathcal{U}_{c, \theta}$ for some $c>0$, and $\theta \in \mathcal{K}_{\infty}$

Corollary 1 (Inputs with bounded variation) Suppose that the hypotheses of Theorem 1 hold with $a<0$ in (7b). Then, system (5) is uniformly asymptotically stable over $\mathcal{U}_{M}$.

Proof To prove the desired result, we derive the constant $c$ and function $\theta$ in (8). Letting $b:=\max \left\{\bar{b}_{i},-\underline{b}_{i}\right\}$, we note that

$$
\begin{aligned}
\bar{b}_{j} \mu_{j}^{+}\left(\left[t_{0}, t\right]\right)-\underline{b}_{j} \mu_{j}^{-}\left(\left[t_{0}, t\right]\right) & \leq b\left(\mu_{j}^{+}\left(\left[t_{0}, t\right]\right)+\mu_{j}^{-}\left(\left[t_{0}, t\right]\right)\right) \\
& =b\left|\mu_{j}\right|_{\left[t_{0}, t\right]} .
\end{aligned}
$$

Since the total variation is non-decreasing with $t$ and is bounded by $M$ for all $t \geq t_{0}$, we can choose $c:=b M$ and $\theta(r):=-a r$ to see that the inequality (8) indeed holds.

For the case $a>0$, it is seen that (8) would not hold for $u \in \mathcal{U}_{M}$ because the contribution of the input $u(\cdot)$ on the left hand side is bounded by a constant as shown in (13) whereas the term $a\left(t-t_{0}\right)$ grows unbounded; and hence the sum cannot be bounded by a decreasing function of time.

\subsection{Switched systems}

Consider an autonomous switched nonlinear system:

$$
\dot{x}(t)=g_{\sigma(t)}(x(t))
$$

where $\sigma(t) \in\{1, \ldots, m\}$ is a right-continuous piecewise-constant switching signal that determines the active vector field $g_{\sigma(t)}(x(t))$ at any given time instant. It is possible to write system (15) in the form of (5). For that, introduce the functions $\chi_{j}(\cdot), j=1, \ldots, m$, as follows:

$$
\chi_{j}(t)= \begin{cases}1 & \text { if } \sigma(t)=j \\ 0 & \text { if } \sigma(t) \neq j\end{cases}
$$

and let $u_{j}(t)=\int_{t_{0}}^{t} \chi_{j}(s) d s$. For such an input, $\mu(\{t\})=0$ for all $t \in\left[t_{0}, \infty\right)$ and one may set $u^{-}(t) \equiv 0$. Then system (15) could be equivalently written as

$$
\dot{x}(t)=\sum_{j=1}^{m} g_{j}(x(t)) d u_{j}
$$

where $d u_{j}=\chi_{j}(\cdot), j=1, \ldots, m$. It is entirely possible that the flow of the state trajectories decays along certain vector fields (called stable), while it 
grows along the other vector fields (called unstable). Intuitively speaking, if there is a certain bound on how long the unstable vector fields are active, then all the state trajectories should still converge to the origin in the long run. Such results with constraints on activation rate of unstable vector fields have appeared in [30]. We show that such rate constraints on the switching signal lead to condition (8) and could be stated as a corollary of Theorem 1.

Towards that end, let us split the set $\{1, \ldots, m\}$ into a disjoint union of two sets $\Sigma_{\mathrm{s}}$ and $\Sigma_{\mathrm{us}}$, such that $g_{j}(\cdot), j \in \Sigma_{\mathrm{s}}$, are the stable vector fields and $\Sigma_{\text {us }}$ indexes the unstable vector fields. The activation time of unstable vector fields over an interval $I$ is denoted by $\mu_{\mathrm{us}}(I):=\sum_{j \in \Sigma_{\mathrm{us}}} \mu_{j}(I)$, and that of stable vector fields is denoted by $\mu_{\mathrm{s}}(I):=\sum_{j \in \Sigma_{\mathrm{s}}} \mu_{j}(I)$.

Corollary 2 For system (15), suppose there exists a continuously differentiable function $V(\cdot)$ that satisfies (7a), and in addition there exist some constants $b_{\mathrm{s}}, b_{\mathrm{us}}>0$ such that

$$
\begin{array}{ll}
\left\langle\nabla V(x), g_{j}(x)\right\rangle \leq-b_{\mathrm{s}} V(x), \quad & \forall x \in \mathbb{R}^{n}, \quad j \in \Sigma_{\mathrm{s}} \\
\left\langle\nabla V(x), g_{j}(x)\right\rangle \leq b_{\mathrm{us}} V(x), \quad \forall x \in \mathbb{R}^{n}, \quad j \in \Sigma_{\mathrm{us}} .
\end{array}
$$

If there exist some finite $r, T>0$ such that the switching signal $\sigma(\cdot)$ satisfies

$$
\frac{1}{T} \mu_{\mathrm{us}}([t, t+T]) \leq r \leq \frac{b_{\mathrm{s}}-\bar{\theta}}{b_{\mathrm{s}}+b_{\mathrm{us}}}, \quad \forall t \geq t_{0}
$$

for some constant $0<\bar{\theta}<b_{\mathrm{s}}$, then the system (15) is globally asymptotically stable.

Proof For any $t \geq t_{0}$, we can write $t=t_{0}+s+\left\lfloor\frac{t-t_{0}}{T}\right\rfloor T$, where $0<s<T$. Under condition $(16), \mu_{\mathrm{us}}\left(\left[t_{0}, t\right]\right) \leq r T+r T\left\lfloor\frac{t-t_{0}}{T}\right\rfloor$ and $\mu_{\mathrm{s}}\left(\left[t_{0}, t\right]\right) \geq(1-r) T\left\lfloor\frac{t-t_{0}}{T}\right\rfloor-$ $r T$, and hence

$$
\begin{aligned}
b_{\mathrm{us}} \mu_{\mathrm{us}}\left(\left[t_{0}, t\right]\right)-b_{\mathrm{s}} \mu_{\mathrm{s}}\left(\left[t_{0}, t\right]\right) & \leq\left(b_{\mathrm{us}}+b_{\mathrm{s}}\right) r T+\left(b_{\mathrm{us}} r-(1-r) b_{\mathrm{s}}\right) T\left\lfloor\frac{t-t_{0}}{T}\right\rfloor \\
& \leq\left(b_{\mathrm{us}}+b_{\mathrm{s}}\right) r T-\bar{\theta} T\left\lfloor\frac{t-t_{0}}{T}\right\rfloor .
\end{aligned}
$$

Thus, the condition (8) holds with $c=\left(b_{\mathrm{us}}+b_{\mathrm{s}}\right) r T$ and $\theta \in \mathcal{K}_{\infty}$ defined as $\theta(s):=\bar{\theta} T\left\lfloor\frac{s}{T}\right\rfloor$. The desired result now follows from Theorem 1 .

\subsection{Impulsive systems}

If each component of the input $u(\cdot)$ in $(5)$ is a piecewise-constant function, then the measure $\mu$ is purely atomic and induces jumps in the state trajectories as well. In that case, (5) could be seen as an impulsive system where the continuous flow of the trajectories is obtained by integration of the vector field $f(\cdot)$ and the discrete component is characterized through Proposition 2. In particular, the step inputs lead to the following equivalent description of system (5):

$$
\begin{aligned}
\dot{x}(t) & =f(x(t)) \\
x\left(t_{i}^{+}\right) & =\Phi_{G}\left(1 ; x\left(t_{i}^{-}\right), \mu\left(\left\{t_{i}\right\}\right)\right)
\end{aligned}
$$

where the set $\left\{t_{i}\right\}_{i \in \mathcal{I}}$ denotes the atoms of the measure $\mu$.

When studying stability of impulsive systems, conditions are derived on the vector field $f(\cdot)$ and the jump map $\psi(\cdot)$ which guarantee stability. Theorem 1 
presents a sufficient condition where neither the continuous dynamics, nor the discrete dynamics, are required to be stable. However, some existing results on stability of impulsive systems in terms of conditions on impulse-time sequence could be derived as a particular case of Theorem 1. Towards that end, let $\mathcal{U}_{\text {step }}$ denote the class of piecewise constant non-decreasing inputs and for each $u \in \mathcal{U}_{\text {step }}$, assume that there is a uniform upper and lower bound on the size of jump in each component of $u(\cdot)$, that is, $0 \leq \mu \leq \mu_{j}\left(\left\{t_{i}\right\}\right) \leq \bar{\mu}<\infty$, for $j=1, \ldots, m$ and $i \in \mathcal{I}$. Let $N(t, s)$ denote the number of discontinuities of $u(\cdot)$ over the interval $(t, s)$. If the length of the interval between two consecutive discontinuities of $u \in \mathcal{U}_{\text {step }}$ is at least $\tau_{a}>0$ on the average, then

$$
N(t, s) \leq N_{0}+\frac{t-s}{\tau_{a}}
$$

where $N_{0} \geq 1$ is some chattering bound and $\tau_{a}$ denotes the average dwelltime between the two atoms of $\mu$. We denote by $\mathcal{U}_{\text {avg }}$ the class of piecewise constant inputs for which $N(t, s)$ satisfies the bound (18). Similarly, one can define $\mathcal{U}_{\text {r-avg }}$ to be the class of piecewise constant inputs for which $N(t, s)$ satisfies the following reverse average dwell-time condition:

$$
N(t, s) \geq \frac{t-s}{\tau_{r}}-N_{0}
$$

We arrive at the following corollary, which is essentially in the same spirit as [19, Theorem 1], and also resembles the stability conditions formulated in terms of a comparison system [22].

Corollary 3 Consider system (5) and suppose there exist a continuously differentiable function $V(\cdot)$ that satisfies $(7 \mathrm{a}),(7 \mathrm{~b})$, and constants $b_{j} \in \mathbb{R}$ such that for each $x \in \mathbb{R}^{n}$

$$
\left\langle\nabla V(x), g_{j}(x)\right\rangle \leq b_{j} V(x), \quad j=1, \ldots, m .
$$

Assume that $\mu_{j}\left(\left\{t_{i}\right\}\right) \geq 0$ for each $t_{i} \in\left[t_{0}, \infty\right), j=1, \ldots, m$, and each $u \in$ $\mathcal{U}_{\text {step. }}$. If there exist $c>0$, and a function $\theta \in \mathcal{K}_{\infty}$ that satisfy

$$
\sum_{t_{i} \in\left[t_{0}, t\right]} \sum_{j=1}^{m} b_{j} \mu_{j}\left(\left\{t_{i}\right\}\right)+a\left(t-t_{0}\right) \leq c-\theta\left(t-t_{0}\right), \quad \forall t \geq t_{0},
$$

then system (5) is asymptotically stable over $\mathcal{U}_{\text {step }}$. In particular,

1. if $a<0, b_{\mathrm{us}}:=\max _{j \in\{1, \ldots, m\}} b_{j} \geq 0$, and $\tau_{a}$ satisfies:

$$
\tau_{a} \geq \frac{b_{\mathrm{us}} m \bar{\mu}}{-(a+\bar{\theta})}
$$

for some $0<\bar{\theta}<|a|$, then system (5) is asymptotically stable over $\mathcal{U}_{\text {avg }}$.

2. if $a \geq 0, b_{\mathrm{s}}:=\max _{j \in\{1, \ldots, m\}} b_{j}<0$, and $\tau_{r}$ satisfies:

$$
\tau_{r} \leq \frac{-b_{\mathrm{s}} m \underline{\mu}}{a+\bar{\theta}}
$$

for some $\bar{\theta}>0$, then system (5) is asymptotically stable over $\mathcal{U}_{\text {r-avg }}$. 
Proof Equation (20) is equivalent to (8) for step inputs when $u(\cdot)$ is nondecreasing at jumps. To prove statements 1 and 2 , we note that

$$
m \underline{\mu} N\left(t_{0}, t\right) \leq \sum_{t_{i} \in\left[t_{0}, t\right]} \mu\left(\left\{t_{i}\right\}\right) \leq m \bar{\mu} N\left(t_{0}, t\right) .
$$

For the first statement with $a<0$ and $b_{\mathrm{us}} \geq 0$, using (18), we have

$$
\begin{aligned}
\sum_{t_{i} \in\left[t_{0}, t\right]} \sum_{j=1}^{m} b_{j} \mu_{j}\left(\left\{t_{i}\right\}\right)+a\left(t-t_{0}\right) & \leq b_{\mathrm{us}} m \bar{\mu} N\left(t_{0}, t\right)+a\left(t-t_{0}\right) \\
& \leq b_{\mathrm{us}} m \bar{\mu}\left(N_{0}-\frac{(a+\bar{\theta})\left(t-t_{0}\right)}{b_{\mathrm{us}} m \bar{\mu}}\right)+a\left(t-t_{0}\right) \\
& =b_{\mathrm{us}} m \bar{\mu} N_{0}-\bar{\theta}\left(t-t_{0}\right)
\end{aligned}
$$

which is a particular of (20) with $c=b_{\mathrm{us}} m \bar{\mu} N_{0}$ and $\theta \in \mathcal{K}_{\infty}$ defined as $\theta(s):=\bar{\theta} s$.

For the second statement with $a \geq 0$ and $b_{\mathrm{s}}<0$, (19) leads to

$$
\begin{aligned}
\sum_{t_{i} \in\left[t_{0}, t\right]} \sum_{j=1}^{m} b_{j} \mu_{j}\left(\left\{t_{i}\right\}\right)+a\left(t-t_{0}\right) & \leq b_{\mathrm{s}} m \underline{\mu} N\left(t_{0}, t\right)+a\left(t-t_{0}\right) \\
& \leq b_{\mathrm{s}} m \underline{\mu}\left(\frac{(a+\bar{\theta})\left(t-t_{0}\right)}{-b_{\mathrm{s}} m \underline{\mu}}-N_{0}\right)+a\left(t-t_{0}\right) \\
& =-b_{\mathrm{s}} m \underline{\mu} N_{0}-\bar{\theta}\left(t-t_{0}\right)
\end{aligned}
$$

which is again a particular case of (20) with $c=-b_{\mathrm{s}} m \underline{\mu} N_{0}>0$ and $\theta \in \mathcal{K}_{\infty}$ defined as $\theta(s):=\bar{\theta} s$.

Remark 3 In the light of Proposition 2, discontinuities in the state trajectories of MDEs are basically defined as the solutions of certain differential equations. That is why, without requiring the explicit knowledge of the jump map, we can directly formulate stability conditions in terms of the vector fields whose flow determine the value of state after the jump. This is in contrast to the impulsive ODEs $[17,22,23]$, which in general are modeled as:

$$
\begin{aligned}
\dot{x}(t) & =f(x(t)), \quad t \neq t_{i} \\
x\left(t_{i}^{+}\right) & =\psi\left(x\left(t_{i}^{-}\right)\right) .
\end{aligned}
$$

The equation (21b) is more general than (17b) because not every function $\psi(\cdot)$ satisfies the property of the flow of a vector field. The stability conditions for such impulsive systems are basically obtained by mapping the trajectories of (21) to a scalar system, and then studying the stability properties of that comparison system, which typically require the explicit knowledge of the jump map and depend on how frequently the state resets occur. While in impulsive ODEs, the state reset map is usually fixed, the formalism of MDEs and our stability results can allow for multiple jump maps (just by adding control vector fields with appropriate inputs), and can also incorporate switched dynamics. Also, the well-posedness of system (21) is not guaranteed for arbitrary jump map $\psi(\cdot)$ whereas the use of bounded variation functions to model state trajectories allows us to deduce the existence and uniqueness of solutions under mild assumptions, even in the presence of possible Zeno phenomenon. 


\section{Input-to-State Stability with Variation}

In contrast to asymptotic stability for a specific class of inputs, we are now interested in studying another notion of stability for system (5). The motivation is to consider systems which are not necessarily asymptotically stable but the maximum value of the state trajectories depends on some norm of the driving input. This may be particularly interesting when $u(\cdot)$ models additive noise or there are undesired impulsive perturbations in the state trajectory.

Definition 3 System (5) is called input-to-state stable (ISS) with respect to variation of the input $u(\cdot)$ if there exist a class $\mathcal{K} \mathcal{L}$ function $\beta(\cdot, \cdot)$ and a class $\mathcal{K}_{\infty}$ function $\gamma(\cdot)$ such that

$$
|x(t)| \leq \beta\left(\left|x\left(t_{0}\right)\right|, t-t_{0}\right)+\gamma\left(|\mu|_{\left[t_{0}, t\right]}\right) \quad t \geq t_{0} .
$$

Next, we present sufficient conditions under which the property (22) holds.

Theorem 2 (ISS with Variation) Suppose that there exist a continuously differentiable function $V: \mathbb{R}^{n} \rightarrow \mathbb{R}_{+}$, some class $\mathcal{K}_{\infty}$ functions $\alpha_{1}(\cdot), \alpha_{2}(\cdot)$, and some positive constants $a, b, c>0$ such that for each $x \in \mathbb{R}^{n}$ :

$$
\begin{aligned}
\alpha_{1}(|x|) \leq V(x) & \leq \alpha_{2}(|x|), \\
\langle\nabla V(x), f(x)\rangle & \leq-a V(x), \\
-b V(x)-c \leq\left\langle\nabla V(x), g_{i}(x)\right\rangle & \leq b V(x)+c, \quad i=1, \ldots, m,
\end{aligned}
$$

then system (5) is ISS with respect to the variation of $u(\cdot)$.

Proof Consider the composition function $W: \mathbb{R} \rightarrow \mathbb{R}_{+}$defined as $t \rightarrow V(x(t))$, which is also locally $R C B V$ by the same reasoning given in the proof of Theorem 1 . Over an interval $\left(t_{i}, t_{i+1}\right)$ between two discontinuities of the input $u(\cdot)$, we have

$$
\begin{aligned}
d W & =\langle\nabla V(x), f(x)\rangle d t+\sum_{j=1}^{m}\left\langle\nabla V(x), g_{j}(x)\right\rangle d u_{j} \\
& \leq-a W d t+b W \sum_{j=1}^{m} d u_{j}+c \sum_{j=1}^{m} d u_{j} .
\end{aligned}
$$

Using Lemma 1 in Appendix B, the following holds for each $t \in\left(t_{i}, t_{i+1}\right)$ :

$$
W(t) \leq e^{-a\left(t-t_{i}\right)+b|\mu|_{\left[t_{i}, t\right)}} W\left(t_{i}\right)+c e^{b|\mu|_{\left[t_{i}, t\right)}}|\mu|_{\left[t_{i}, t\right)} .
$$

To obtain an upper bound on $W\left(t_{i}^{+}\right)$at a jump instant $t_{i}$, we follow the same arguments as in the proof of Theorem 1. Letting $z_{i}(\cdot)$ denote the solution of (10), we have $\dot{V}\left(z_{i}(s)\right) \leq b V\left(z_{i}(s)\right)+c$ for $s \in[0,1]$ using (23c), and the comparison lemma yields:

$$
V(z(1)) \leq e^{b|\mu|_{\left\{t_{i}\right\}}} V(z(0))+\frac{c}{b}\left(e^{b|\mu|_{\left\{t_{i}\right\}}}-1\right)
$$

where we recall that $|\mu|_{\left\{t_{i}\right\}}=\mu^{+}\left(\left\{t_{i}\right\}\right)+\mu^{-}\left(\left\{t_{i}\right\}\right)$. Using the fact that $z_{i}(0)=$ $x\left(t_{i}^{-}\right)$, and $z_{i}(1)=x\left(t_{i}^{+}\right)$, the above inequality gives

$$
W\left(t_{i}^{+}\right) \leq e^{b|\mu|_{\left\{t_{i}\right\}}} W\left(t_{i}^{-}\right)+\frac{c}{b}\left(e^{b|\mu|_{\left\{t_{i}\right\}}}-1\right) .
$$


Using these expressions for continuous and discrete flow of the function $W(\cdot)$, it is observed that for any $t \in\left(t_{i}, t_{i+1}\right), i \geq 1$, we have

$$
W(t) \leq W_{i c}(t)+W_{u_{c}}(t)+W_{u_{d}}(t)
$$

where $W_{i c}(\cdot)$ denotes the evolution of $W(\cdot)$ due to the initial condition $W\left(t_{0}\right)$, $W_{u_{c}}(\cdot)$ denotes the evolution due to continuous part of the input between discontinuities, and $W_{u_{d}}$ denotes the evolution due to jumps in the input. The value of these three functions is given by:

$$
\begin{gathered}
W_{i c}(t)=e^{-a\left(t-t_{0}\right)+b|\mu|_{\left[t_{0}, t\right)} W\left(t_{0}\right)} \\
W_{u_{c}}(t)=c \sum_{k=0}^{i-1} e^{-a\left(t-t_{k+1}\right)+b|\mu|_{\left(t_{k}, t\right)}|\mu|_{\left(t_{k}, t_{k+1}\right)}+c e^{b|\mu|_{\left(t_{i}, t\right)}|\mu|_{\left(t_{i}, t\right)}}} \\
W_{u_{d}}(t)=\frac{c}{b} \sum_{k=1}^{i} e^{-a\left(t-t_{k}\right)+b|\mu|_{\left(t_{k}, t\right)}}\left(e^{b|\mu|_{\left\{t_{k}\right\}}}-1\right) .
\end{gathered}
$$

Using $e^{-a\left(t-t_{k}\right)} \leq 1$ and $e^{b|\mu|_{\left(t_{k}, t\right)}} \leq e^{b|\mu|_{\left[t_{0}, t\right]}}$ for each $k \leq i$, we obtain

$$
W_{u_{d}} \leq \frac{c}{b} e^{b|\mu|_{\left[t_{0}, t\right]}} \sum_{k=1}^{i}\left(e^{b|\mu|_{\left\{t_{k}\right\}}}-1\right)
$$

To obtain similar a bound for $W_{u_{c}}(t)$, we multiply and divide (25) by $b$, and in addition use the fact $r \leq e^{r}-1$, for $r \geq 0$, to get

$$
W_{u_{c}}(t) \leq \frac{c}{b} e^{b|\mu|_{\left[t_{0}, t\right]}}\left(\sum_{k=0}^{i-1}\left(e^{b|\mu|_{\left(t_{k}, t_{k+1}\right)}}-1\right)+\left(e^{b|\mu|_{\left(t_{i}, t\right)}}-1\right)\right) .
$$

Adding (26) and (27), and using the fact $\left(e^{r_{1}}-1\right)+\left(e^{r_{2}}-1\right) \leq e^{r_{1}+r_{2}}-1$, we have

$$
W_{u_{c}}(t)+W_{u_{d}}(t) \leq \frac{c}{b} e^{b|\mu|_{\left[t_{0}, t\right]}}\left(e^{b|\mu|_{\left[t_{0}, t\right]}}-1\right) .
$$

Finally, the upper bound on $W_{i c}(t)$ is obtained as follows:

$$
\begin{aligned}
W_{i c}(t) & =e^{-a\left(t-t_{0}\right)} W\left(t_{0}\right)+e^{-a\left(t-t_{0}\right)}\left(e^{b|\mu|_{\left[t_{0}, t\right]}}-1\right) W\left(t_{0}\right) \\
& \leq e^{-a\left(t-t_{0}\right)} W\left(t_{0}\right)+\frac{1}{2}\left(e^{-a\left(t-t_{0}\right)} W\left(t_{0}\right)\right)^{2}+\frac{1}{2}\left(e^{b|\mu|_{\left[t_{0}, t\right]}}-1\right)^{2} .
\end{aligned}
$$

The estimate of the form (22) can now be computed by introducing the following functions:

$$
\beta(r, t):=\alpha_{1}^{-1}\left(\hat{\theta}\left(e^{-a t} \alpha_{2}(r)\right)\right)
$$

where $\hat{\theta}(r):=r+\frac{1}{2} r^{2}$ is a class $\mathcal{K}_{\infty}$ function, and

$$
\gamma(s):=\frac{1}{2}\left(e^{b s}-1\right)^{2}+\frac{c}{b} e^{b s}\left(e^{b s}-1\right) .
$$

Plugging (28), (29) in (24) and noting that $W\left(x\left(t_{0}\right)\right) \leq \alpha_{2}\left(\left|x\left(t_{0}\right)\right|\right)$ and $|x(t)| \leq$ $\alpha_{1}^{-1}(W(x(t)))$, the desired result $(22)$ is obtained with $\beta(\cdot)$ and $\gamma(\cdot)$ defined in (30) and (31), respectively. 
Remark 4 For an absolutely continuous function $u(\cdot)$, the variation of $u(\cdot)$ is equal to the $\mathcal{L}_{1}$-norm of $\dot{u}(\cdot)$. Since system (5), in that case, could be thought of as driven by the derivative of $u(\cdot)$, the notion of ISS given in Definition 3 coincides with the notion of integral ISS (discussed in [39]) with respect to $\dot{u}(\cdot)$. Comparing our sufficient conditions with the ones presented in that paper, we chose to work with a linear multiple of the norm of the input instead of an arbitrary class $\mathcal{K}_{\infty}$ function so that the resulting inequalities involving measures could be explicitly solved.

Example 3 Let us recall the example considered in Section 1.3 with MDE $d x=-x d t+d u$. We pick the Lyapunov function to be $V(x)=\frac{1}{2} x^{2}$. It is easy to see that the inequalities (23) hold with $\alpha_{1}(r)=\alpha_{2}(r)=\frac{1}{2} r^{2}$, and the constants $a=b=2, c=1$. Thus the system is ISS with respect to variation.

Example 4 Consider the following bilinear system:

$$
d x=A x d t+\left(B_{1} x+B_{2}\right) d u
$$

with $A$ being a Hurwitz matrix. We show that this system is ISS with respect to variation of the input. To see this, we consider the Lyapunov function $V=x^{\top} P x$ where $P$ is a symmetric positive definite matrix satisfying $A^{\top} P+$ $P A=-a P$ for some $a>0$. Thus, (23a) and (23b) hold. To find the desired constants in $(23 \mathrm{c})$, note that

$$
\left\langle 2 P x, B_{1} x\right\rangle \leq 2\left\|P B_{1}\right\| \cdot|x|^{2}
$$

where $\left\|P B_{1}\right\|$ denotes the induced Euclidean norm of the matrix $P B_{1}$. Also,

$$
\left\langle 2 P x, B_{2}\right\rangle \leq 2\left\|P B_{2}\right\| \cdot|x| \leq\left\|P B_{2}\right\|^{2}+|x|^{2}
$$

Similarly, by using the Cauchy-Schwarz inequality in other direction, we get

$$
\left\langle 2 P x, B_{1} x+B_{2}\right\rangle \geq-\left(2\left\|P B_{1}\right\|+1\right)|x|^{2}-\left\|P B_{2}\right\|^{2} .
$$

Using the fact $|x|^{2} \leq \frac{1}{\lambda_{\min }(P)} V(x)$, it is observed that (23c) holds with

$$
b=\frac{2\left\|P B_{1}\right\|+1}{\lambda_{\min }(P)}, \quad c=\left\|P B_{2}\right\|^{2} .
$$

\section{Conclusion}

In this paper, we studied the problem of stability in a class of differential equations driven linearly by differential measures associated with functions of locally bounded variation. Sufficient conditions based on Lyapunov function were given for uniform asymptotic stability over a certain class of inputs. We then studied a generalized notion of integral ISS in measure driven systems and presented sufficient conditions under which the supremum norm of the state is bounded by the total variation of the measure associated with input. 


\section{A Solution of MDEs}

We study the existence of solution of system (5) and the content of this appendix is primarily borrowed from [12]. Before arriving at the main result, we first recall a definition that is essential for the development to follow.

Definition 4 Let $u:\left[t_{0}, T\right] \rightarrow R^{m}$ be an $R C B V$ function and set

$$
U(t):=\frac{t-t_{0}+\operatorname{var}_{u}\left(t_{0} ; t\right)}{T-t_{0}+\operatorname{var}_{u}\left(t_{0} ; T\right)}, \quad t \in\left[t_{0}, T\right] .
$$

The canonical graph completion $\varphi:[0,1] \rightarrow\left[t_{0}, T\right] \times \mathbb{R}^{m}$ of $u(\cdot)$ is defined as:

$$
\varphi(s):= \begin{cases}(t, u(t)) & \text { if } s=U(t) \\ \left(t, u(t)+\frac{s-U(t)}{U\left(t^{+}\right)-U\left(t^{-}\right)} u\left(t^{+}\right)-u\left(t^{-}\right)\right) & \text {if } s \in\left(U\left(t^{-}\right), U\left(t^{+}\right)\right) .\end{cases}
$$

We now define a coordinate transformation using the canonical graph completion of the input. Let $\varphi:=\left(\varphi_{0}, \varphi_{1}, \ldots, \varphi_{m}\right)$ denote the graph completion of $u(\cdot)$ and consider the following auxiliary $(n+1)$ dimensional, Cauchy problem

$$
\begin{aligned}
\frac{d y_{0}}{d s} & =\frac{d \varphi_{0}}{d s}, \quad y_{0}(0)=0 \\
\frac{d y}{d s} & =f(y(s)) \frac{d \varphi_{0}}{d s}+\sum_{j=1}^{m} g_{j}(y(s)) \frac{d \varphi_{j}}{d s}, \quad y(0)=x_{0}
\end{aligned}
$$

where $\tilde{y}:=\left(y_{0}, y\right):[0,1] \rightarrow[0, T] \times \mathbb{R}^{n}$. Since the graph completions (by definition) are Lipschitz continuous functions, the solutions of (32) are studied in the classical sense. The following result establishes the link between the solutions of auxiliary system (32) and (6).

Theorem 3 ([12, Theorem 2.2]) Let $u:\left[t_{0}, T\right] \rightarrow \mathbb{R}^{m}$ be an $R C B V$ function. An $R C B V$ function $x(\cdot)$ is called a solution of (6) if and only if there exists a solution $\tilde{y}=\left(y_{0}, y\right)$ of (32) corresponding to the canonical graph completion $\varphi(\cdot)$ such that

$$
x(t)=y(U(t))
$$

for almost every $t \in(0, T)$.

A straightforward consequence of this result is that there exists a unique solution to system (5) whenever there exists a unique solution to system (32) in the classical sense. For scalar inputs, it is important to note that the forward completeness of the vector fields $f(\cdot)$ and $g(\cdot)$ is not sufficient to guarantee that the solution of $(32 \mathrm{~b})$ is forward complete. While working with continuous inputs, a counterexample has been given in [40, Section 8]. In the same paper in Theorem 2, were presented sufficient conditions (which roughly state that $f(\cdot)$ satisfies a linear growth condition and $g(\cdot) \in \mathcal{C}^{1}\left(\mathbb{R}^{n}, \mathbb{R}^{n}\right)$ with $\frac{\partial g}{\partial x}$ uniformly bounded) that guarantee the existence and uniqueness of the solutions to (32) with $u(\cdot)$ being continuous. Later, it was shown in [5, Theorem 2.1] that these conditions are sufficient for existence of global (in time) solutions of (32b). Without imposing such strong conditions on system vector fields, it is nonetheless assumed in this paper that there exists a unique solution to system (32) for every Lipschitz continuous function $\varphi(\cdot)$.
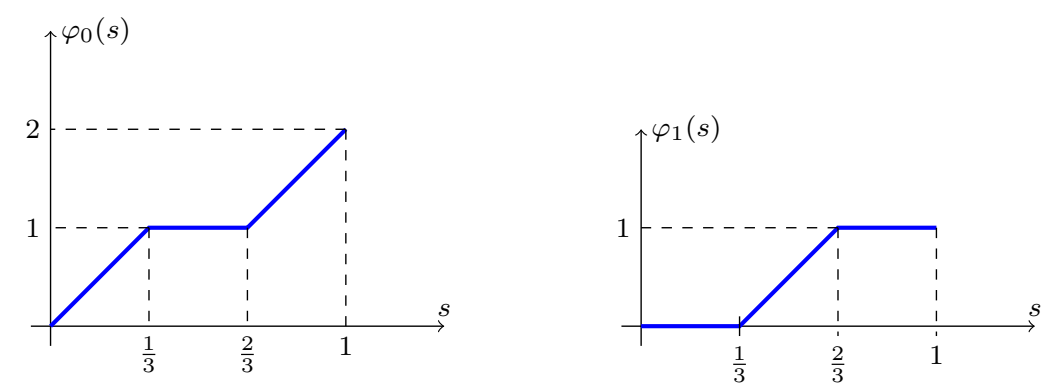

Fig. 1 Canonical graph completion of a step function $u:[0,2] \rightarrow\{0,1\}$ with $u(t)=0$ for $0 \leq t<1$, and $u(t)=1$ for $1 \leq t \leq 2$. 


\section{B Generalized Comparison Lemma}

Lemma 1 (Comparison lemma for MDEs) Consider a continuous locally BV function $u:\left(t_{0}, t_{1}\right) \rightarrow \mathbb{R}^{m}$ with du as the associated differential measure and a continuous function $V:\left(t_{0}, t_{1}\right) \rightarrow \mathbb{R}$ that satisfies

$$
d V \leq-a V d t+b V \sum_{j=1}^{m} d u_{j}+c \sum_{j=1}^{m} d u_{j}
$$

then for each $t \in\left(t_{0}, t_{1}\right)$

$$
V(t) \leq e^{-a\left(t-t_{0}\right)+b \sum_{j=1}^{m} \mu_{j}\left(\left(t_{0}, t\right)\right)} V\left(t_{0}^{+}\right)+c e^{b|\mu|_{\left(t_{0}, t\right)}}|\mu|_{\left(t_{0}, t\right)}
$$

where $\mu$ is the Lebesgue-Stieltjes measure associated with $u(\cdot)$.

Proof We split the proof in two steps:

Step 1. Consider a right-continuous function $W:\left(t_{0}, t_{1}\right) \rightarrow \mathbb{R}$ such that

$$
d W=-a W d t+b W \sum_{j=1}^{m} d u_{j}+c \sum_{j=1}^{m} d u_{j}
$$

with $W\left(t_{0}^{+}\right)=V\left(t_{0}^{+}\right)$. Let us consider a sequence $\left\{u^{k}\right\}_{k=1}^{\infty}$ of continuously differentiable functions converging pointwise to $u(\cdot)$ and having the property that

$$
\lim _{k \rightarrow \infty} \operatorname{var}_{u_{k}}\left(t_{0}^{+} ; t_{1}^{-}\right)=\operatorname{var}_{u}\left(t_{0}^{+} ; t_{1}^{-}\right) .
$$

Let $W^{k}(\cdot)$ represent the solution of (34) obtained by replacing $d u_{j}$ with $\dot{u}_{j}^{k} d t, j=1, \ldots, m$. Then

$$
W^{k}(t)=\Psi\left(t, t_{0}\right) W^{k}\left(t_{0}\right)+c \sum_{j=1}^{m} \int_{t_{0}}^{t} \Psi(t, s) \dot{u}_{j}^{k}(s) d s
$$

where $\Psi(t, s)=e^{-a(t-s)+b \sum_{j=1}^{m}\left(u_{j}^{k}(t)-u_{j}^{k}(s)\right)}$ and $W^{k}\left(t_{0}^{+}\right)=W\left(t_{0}^{+}\right)$for each $k \geq 1$. Using the inequalities $e^{-a(t-s)} \leq 1$ and $\left|u^{k}(t)-u^{k}(s)\right|_{1} \leq \int_{t_{0}}^{t}\left|\dot{u}^{k}(\tau)\right|_{1} d \tau$ for each $s \in\left(t_{0}, t\right)$, $t<t_{1}$, we obtain

$$
W^{k}(t) \leq e^{-a\left(t-t_{0}\right)+b \sum_{j=1}^{m}\left(u_{j}^{k}(t)-u_{j}^{k}\left(t_{0}\right)\right)} W^{k}\left(t_{0}\right)+c e^{b\left\|\dot{u}_{\left(t_{0}, t\right)}^{k}\right\|_{1}}\left\|\dot{u}_{\left(t_{0}, t\right)}^{k}\right\|_{1} .
$$

Noting that, $\left\|\dot{u}_{\left(t_{0}, t\right)}^{k}\right\|_{1}$ denotes the total variation of $\dot{u}_{k}(\cdot)$ over the interval $\left(t_{0}, t\right)$, so by construction $\left\|\dot{u}_{\left(t_{0}, t\right)}^{k}\right\|_{1}$ converges to $|\mu|_{\left(t_{0}, t\right)}$. Hence,

$$
W(t) \leq e^{-a\left(t-t_{0}\right)+b \sum_{j=1}^{m} \mu_{j}\left(\left(t_{0}, t\right)\right)} W\left(t_{0}^{+}\right)+c e^{b|\mu|_{\left(t_{0}, t\right)}}|\mu|_{\left(t_{0}, t\right)} .
$$

Step 2. We now show that $V(t) \leq W(t)$ for each $t \in\left(t_{0}, t_{1}\right)$, from which the desired result follows. Indeed, if $V$ and $W$ satisfy (33) and (34), then $\bar{W}:=V-W$ satisfies

$$
d \bar{W} \leq-a \bar{W} d t+b \bar{W} d u
$$

where $\bar{W}\left(t_{0}^{+}\right)=0$. Using the same arguments as in the proof of Theorem 1 , we obtain

$$
\bar{W}(t) \leq e^{-a\left(t-t_{0}\right)+b \mu\left(\left(t_{0}, t\right)\right)} \bar{W}\left(t_{0}^{+}\right)
$$

so that $\bar{W}(t) \leq 0$, or equivalently $V(t) \leq W(t)$

\section{References}

1. V. Acary, B. Brogliato, and D. Goeleven. Higher order Moreau's sweeping process: Mathematical formulation and numerical simulation. Mathematical Programming Ser. A, 113:133 - 217, 2008.

2. L. Ambrosio, N. Fusco, and D. Pallara. Functions of Bounded Variation and Free Discontinuity Problems. Clarendon Press, Oxford, 2000.

3. D. Angeli, E. Sontag, and Y. Wang. A characterization of integral input-to-state stability. IEEE Trans. Automatic Control, 45(6):1082 - 1097, 2000. 
4. A. Bressan. On differential systems with impulsive controls. Rendiconti del Seminario Matematico della Università di Padova, 78:227 - 235, 1987.

5. A. Bressan and F. Rampazzo. On differential systems with quadratic impulses and their applications to Lagrangian mechanics. SIAM J. Control \& Optim., 31(5):1205 - 1220, 1993.

6. A. Bressan Jr. and F. Rampazzo. Impulsive control systems with commutative vector fields. J. Optimization Theory and Applications, 71(1):67-83, 1991.

7. A. Bressan Jr. and F. Rampazzo. Impulsive control systems without commutativity assumptions. J. Optimization Theory and Applications, 81(3):435 - 457, 1994.

8. B. Brogliato. Some results on optimal control with unilateral constraints. Nonlinear Analysis: Theory, Methods, and Applications, 70:3626 - 3657, 2009.

9. T. E. Carter. Optimal impulsive space trajectories based on linear equations. J. Optimization Theory \&3 Applications, 70(2):277 - 297, 1991.

10. M. Claeys, D. Arzelier, D. Henrion, and J.-B. Lasserre. Measures and LMI for impulsive optimal control with applications to space rendezvous problems. In Proc. American Control Conf., pages 161 - 166, Montreal, Canada, 2012.

11. W. J. Code and G. N. Silva. Closed loop stability of measure-driven impulsive control systems. J. Dynamical and Control Systems, 16(1):1 - 21, 2010.

12. G. Dal Maso and F. Rampazzo. On systems of ordinary differential equations with measures as controls. J. Differential \& Integral Equations, 4(4):739 - 765, 1991.

13. P. C. Das and R. R. Sharma. Existence and stability of measure differential equations. Czechoslovak Mathematical Journal, 22(1):145 - 158, 1972.

14. S. Dashkovskiy and A. Mironchenko. Input-to-state stability of nonlinear impulsive systems. SIAM J. Control \& Optim., 51(3):1962 - 1987, 2013.

15. V. A. Dykhta. Impulsive optimal control in models of economics and quantum electronics (in russian). Avtomatika i Telemekhanika, (11):100 - 112, 1999.

16. G. Feichtinger, R. F. Hartl, and S. P. Sethi. Dynamic optimal control models in advertising: Recent developments. J. Management Science, 40(2):195 - 226, 1994

17. W. M. Haddad, V. S. Chellaboina, and S. G. Nersesov. Impulsive and hybrid dynamical systems. Princeton Series in Applied Mathematics. Princeton University Press, Princeton, NJ, 2006.

18. O. Hájek. Book review: Differential systems involving impulses. Bulletin of American Mathematical Society, 12:272 - 279, 1985.

19. J. P. Hespanha, D. Liberzon, and A. Teel. Lyapunov conditions for input-to-state stability of impulsive systems. Automatica, 44:2735 - 2744, 2008.

20. H. K. Khalil. Nonlinear Systems. Prentice-Hall, Inc., 3rd edition, 2002.

21. J. Kurzweil. Generalized ordinary differential equations. Czechoslovak Mathematical Journal, 8(3):360 - 388, 1958.

22. V. Lakshmikantham, D. D. Bainov, and P. S . Simeonov. Theory of Impulsive Differential Equations, volume 6 of Series in Modern Applied Mathematics. World Scientific, Singapore, 1989

23. V. Lakshmikantham and X. Liu. Stability criteria for impulsive differential equations in terms of two measures. Journal of Mathematical Analysis and Applications, 137:591 $-604,1989$.

24. S. Leela. Stability of measure differential equations. Pacific Journal of Mathematics, 55(2):489 - 498, 1974.

25. J. Liu, X. Liu, and W.-C. Xie. Input-to-state stability of impulsive and switching hybrid systems with time-delay. Automatica, 47(5):899 - 908, 2011.

26. B. M. Miller. The generalized solutions of nonlinear optimization problems with impulse control. SIAM J. Control \& Optim., 34(4):1420 - 1440, 1996.

27. M. D. P. Monteiro Marques. Differential Inclusions in Nonsmooth Mechanical Problems: Shocks and Dry Friction, volume 9 of Progress in Nonlinear Differential Equations and their Applications. Birkhäuser, 1993.

28. J. J. Moreau. Bounded variation in time. In J. J. Moreau, P. D. Panagiotopoulos, and G. Strang, editors, Topics in Nonsmooth Mechanics. Birkhäuser Verlag, 1988.

29. J. J. Moreau and M. Valadier. A chain rule involving vector functions of bounded variation. Journal of Functional Analysis, 74(2):333 - 345, 1987.

30. D. Munoz de la Pena and P. D. Christofides. Stability of nonlinear asynchronous systems. In Proc. 46th IEEE Conf. Decision \& Control, pages 4576 - 4583, New Orleans (LA), USA, December 2007.

31. L. W. Neustadt. A general theory of minimum-fuel space trajectories. SIAM J. Control, ser. A, 3(2):317 - 356, 1965

32. Y. Orlov. Schwartz' distributions in nonlinear setting: Applications to differential equations, filtering and optimal control. Math. Problems in Engineering, 8(4 - 5):367 - 387, 2002. 
33. S. G. Pandit and S. G. Deo. Differential Systems Involving Impulses, volume 954 of Lecture Notes in Mathematics. Springer-Verlag, 1982.

34. R. W. Rishel. An extended Pontryagin principle for control systems whose control laws contain measures. SIAM J. Control, ser. A, 3(2):191-205, 1965.

35. H. Schaub and K. T. Alfriend. Impulsive feedback control to establish specific mean orbit elements of spacecraft formations. J. Guidance, Navigation $\&$ Control, 24(4):739 $-745,2001$.

36. W. W. Schmaedeke. Optimal control theory for nonlinear vector differential equations containing measures. SIAM J. Control, 3(2):231 - 280, 1965.

37. G. N. Silva and R. B. Vinter. Necessary conditions for optimal impulsive control problems. SIAM J. Control \& Optim., 35(6):1829 - 1846, 1997.

38. E. D. Sontag. Smooth stabilization implies coprime factorization. IEEE Trans. on Automatic Control, AC-34(4):435 - 443, 1989.

39. E. D. Sontag. Comments on integral variants of ISS. Systems and Control Letters, 34:93-100, 1998.

40. H. J. Sussmann. On the gap between deterministic and stochastic ordinary differential equations. The Annals of Probability, 6(1):19-41, 1978.

41. N. van de Wouw and R. I. Leine. Robust impulsive control of motion systems with uncertain friction. Int. J. Robust 83 Nonlinear Control, 22(4):369 - 397, 2012

42. J. Warga. Extended problems with unbounded controls. SIAM J. Control, ser. A, $3(3): 424-438,1966$.

43. Y. Xiaoa, D. Chen, and H. Qin. Optimal impulsive control in periodic ecosystem. Systems \& Control Letters, 55(7):558 - 565, 2006.

44. X. Zhang, Z. Shuai, and K. Wang. Optimal impulsive harvesting policy for single population. Nonlinear Analysis: Real World Applications, 4(4):639 - 651, 2004. 